\title{
Effective action for the Abelian-Higgs model for a gauge-invariant implementation on optical lattices
}

\author{
Alexei Bazavov ${ }^{\star a, b}$, Yannick Meurice $^{b}$, Shan-Wen Tsai ${ }^{a}$, Judah Unmuth-Yockey ${ }^{b}$, Jin \\ Zhang $^{a}$ \\ ${ }^{a}$ Department of Physics and Astronomy, University of California, Riverside, CA 92521, USA \\ ${ }^{b}$ Department of Physics and Astronomy, University of Iowa, Iowa City, IA 52242, USA \\ E-mail: obazavovequark.phy.bnl.gov
}

\begin{abstract}
We present a gauge-invariant effective action for the Abelian-Higgs model in $1+1$ dimensions. It is constructed by integrating out the gauge field and then using the hopping parameter expansion. The latter is tested with Monte Carlo simulations for small values of the scalar self-coupling. In the opposite limit, at infinitely large self-coupling, the Higgs mode is frozen and the partition function can be written in terms of local tensors and the tensor renormalization group blocking can be applied. The numerical implementation requires truncations and the time continuum limit of the blocked transfer matrix can be obtained numerically. At zero gauge coupling and with a spin-1 truncation, the small volume energy spectrum is identical to the low energy spectrum of a two-species Bose-Hubbard model in the limit of large onsite repulsion. The procedure is extended to finite gauge coupling and we derive a spin- 1 approximation of the Hamiltonian which involves terms corresponding to transitions among the two species in the Bose-Hubbard model. An optical lattice implementation involving a ladder structure is proposed.
\end{abstract}

The 33rd International Symposium on Lattice Field Theory

14 - 18 July 2015

Kobe International Conference Center, Kobe, Japan

\footnotetext{
* Speaker.
} 


\section{Introduction}

There has been a lot of interest recently in the possibility of building quantum simulators for lattice gauge theory using optical lattices $[1,2]$. When gauge fields are mapped onto the physical degrees of freedom, such as individual atoms or condensates trapped in an optical lattice, one needs to ensure that the physical observables are gauge invariant. This can be achieved by imposing Gauss's law (which has often to be done approximately) or by constructing an explicitly gauge invariant formulation of the original model. We report on our recent progress in following the latter approach [3].

We establish an approximate quantitative correspondence between the Abelian-Higgs model (scalar electrodynamics) on a 1+1 dimensional lattice and a two-species Bose-Hubbard model with specifically chosen interactions that can in principle be realized experimentally on optical lattices. We derive the gauge-invariant effective action by integrating over the gauge fields. In this case the constraint enforcing gauge invariance is satisfied automatically.

\section{The Abelian-Higgs model and the effective action}

We consider the Abelian-Higgs model on a 1+1 space-time lattice of size $N_{s} \times N_{\tau}$. The scalar field $\phi_{x}=\left|\phi_{x}\right| \exp \left(i \theta_{x}\right)$ is attached to the sites and the gauge fields $U_{x, \hat{v}}=\exp \left(i A_{x, \hat{v}}\right)$ to the links of the lattice. The product of $U$ 's around a plaquette is denoted $U_{p l, x}$ where $x$ is the lower left corner of the plaquette in space-time coordinates. $\beta_{p l}=1 / g^{2}$ is the inverse gauge coupling and $\kappa_{s}\left(\kappa_{\tau}\right)$ is the hopping coefficient in the space (time) direction. The partition function and the action are:

$$
\begin{aligned}
Z & =\int D \phi^{\dagger} D \phi D U e^{-S}, \quad S=-\beta_{p l} \sum_{x} \operatorname{Re}\left[U_{p l, x}\right]+\sum_{x} \phi_{x}^{\dagger} \phi_{x}+\lambda \sum_{x}\left(\phi_{x}^{\dagger} \phi_{x}-1\right)^{2} \\
& -\kappa_{\tau} \sum_{x}\left[\mathrm{e}^{\mu} \phi_{x}^{\dagger} U_{x, \hat{\tau}} \phi_{x+\hat{\tau}}+\mathrm{e}^{-\mu} \phi_{x+\hat{\tau}}^{\dagger} U_{x, \hat{\tau}}^{\dagger} \phi_{x}\right]-\kappa_{s} \sum_{x}\left[\phi_{x}^{\dagger} U_{x, \hat{s}} \phi_{x+\hat{s}}+\phi_{x+\hat{s}}^{\dagger} U_{x, \hat{s}}^{\dagger} \phi_{x}\right] .
\end{aligned}
$$

The Nambu-Goldstone fields $\theta_{x}$ can be eliminated by a gauge transformation

$$
A_{x, \hat{v}} \rightarrow A_{x, \hat{v}}-\theta_{x+\hat{v}}+\theta_{x}
$$

which leaves the plaquette terms unchanged.

Unlike other approaches, e.g. $[1,2,4,5,6,7,8]$, we will not try to implement the gauge field on the optical lattice, but rather try to implement a gauge-invariant effective action obtained by integrating over the gauge fields. A similar approach is being pursued for theories with fermions [9]. For this purpose, we use the Fourier expansion of the Boltzmann weights in terms of the modified Bessel functions $I_{n}$, for instance,

$$
\begin{aligned}
& \exp \left[2 \kappa_{\tau}\left|\phi_{x}\right|\left|\phi_{x+\hat{\tau}}\right| \cos \left(\theta_{x+\hat{\tau}}-\theta_{x}+A_{x, \hat{\tau}}-i \mu\right)\right] \\
= & \sum_{n=-\infty}^{\infty} I_{n}\left(2 \kappa_{\tau}\left|\phi_{x}\right|\left|\phi_{x+\hat{\tau}}\right|\right) \exp \left[\operatorname{in}\left(\theta_{x+\hat{\tau}}-\theta_{x}+A_{x, \hat{\tau}}-i \mu\right)\right],
\end{aligned}
$$

and similar expressions for the space hopping and the plaquette interactions. We can then collect all the exponentials involving a given $A_{x, \hat{v}}$ and perform the integration over $A_{x, \hat{v}}$. This results in 
Kronecker deltas relating the various Fourier modes. The final result is

$$
e^{-S_{e f f}}=\sum_{\left\{m_{\square}\right\}}\left[\prod_{\square} I_{m_{\square}}\left(\beta_{p l}\right) \prod_{x}\left(I_{n_{x, \hat{s}}}\left(2 \kappa_{s}\left|\phi_{x}\right|\left|\phi_{x+\hat{s}}\right|\right) I_{n_{x, \hat{\tau}}}\left(2 \kappa_{\tau}\left|\phi_{x}\right|\left|\phi_{x+\hat{\tau}}\right|\right) \exp \left(\mu n_{x, \hat{\tau}}\right)\right)\right],
$$

where the link indices $n_{x, \hat{v}}$ are related to the plaquette indices $m_{\square}$ by the rules

$$
n_{x, \hat{s}}=m_{\text {below }}-m_{\text {above }}, \quad n_{x, \hat{\tau}}=m_{\text {right }}-m_{\text {left }}
$$

where the subscripts of $m \mathrm{~s}$ refer to the plaquette location with respect to the link. Eqs. (2.5) guarantee that the link indices automatically satisfy the current conservation imposed by the integration of the $\theta_{x}$ variables, so the $m_{\square}$ act as the dual variables.

Equations (2.5) have simple electromagnetic analogs. First, $n_{x, \hat{\tau}}$ can be interpreted as a charge and $m_{x}$ as an electric field in the spatial direction. With this Minkowskian interpretation, Eq. (2.5) enforces Gauss's law. Second, $n_{x, \hat{v}}$ can be interpreted as a two-dimensional current and $m_{x}$ as a magnetic field normal to the two-dimensional plane. In this Euclidean interpretation, Eqs. (2.5) express the current as the curl of the magnetic field.

At the lowest order of the strong-coupling expansion we have $\beta_{p l}=0$ and from $I_{n}(0)=0$ for $n \neq 0$, we see that all the indices must be zeros. The effect of the plaquette can be restored perturbatively. This can be organized in an expansion in the hopping parameter. In the isotropic case $\kappa_{\tau}=\kappa_{s}=\kappa$ we obtain:

$$
S_{e f f}=\sum_{\langle x y\rangle}\left(-\kappa^{2} M_{x} M_{y}+\frac{1}{4} \kappa^{4}\left(M_{x} M_{y}\right)^{2}\right)-2 \kappa^{4} \frac{I_{1}\left(\beta_{p l}\right)}{I_{0}\left(\beta_{p l}\right)} \sum_{\square(x y z w)} M_{x} M_{y} M_{z} M_{w}+O\left(\kappa^{6}\right),
$$

where $M_{x}=\phi_{x}^{\dagger} \phi_{x}$ is a composite gauge-invariant field.

\section{Monte Carlo calculations}

We consider the action (2.1) for the isotropic case $\kappa_{\tau}=\kappa_{s}=\kappa$. In the $\beta_{p l} \rightarrow \infty$ limit when $U_{x, \hat{v}}=1$, the expectation value of the hopping term $L_{\phi}=\left\langle\operatorname{Re}\left\{\phi_{x}^{\dagger} U_{x, \hat{v}} \phi_{x+\hat{v}}\right\}\right\rangle$ can be calculated with the hopping parameter expansion, for small $\kappa$. It has been derived up to $O\left(\kappa^{5}\right)$ in Ref. [10]. This result can be generalized to $\beta_{p l}<\infty$ by including the appropriate factors of $I_{1}\left(\beta_{p l}\right) / I_{0}\left(\beta_{p l}\right)$ for the diagrams that involve plaquettes.

To check the range of validity of the expansion we perform Monte Carlo simulations at several values of $\beta_{p l}, \kappa$ and $\lambda$ on a $16^{2}$ lattice. To test the $\beta_{p l} \rightarrow \infty$ limit we set $\beta_{p l}=20$ and for $\lambda=0.05$ and 0.1 scan the range of $\kappa \in[0.05,0.30]$. The results for $L_{\phi}$ are shown in Fig. 1 . The lines represent the expansion at two orders. The expansion starts to break down around $\kappa=0.15$ at $O\left(\kappa^{3}\right)$ and $\kappa=0.2$ at $O\left(\kappa^{5}\right)$.

To understand the dependence on $\beta_{p l}$ better we also calculate $L_{\phi}$ for several values of $\beta_{p l}$ at fixed $\kappa=0.15$. The results together with the hopping expansion are shown in Fig. 2. There is good agreement, and the dependence on $\beta$ is weak. 


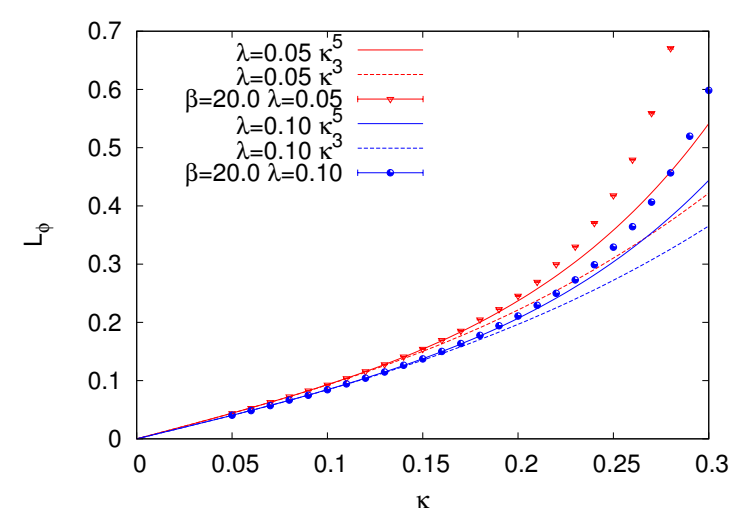

Figure 1: $L_{\phi}$ at $\beta_{p l}=20$ for $\lambda=0.05$ and $\lambda=0.1$ as function of $\kappa$ compared with the hopping expansion at $\beta_{p l}=\infty$ at $O\left(\kappa^{3}\right)$ and $O\left(\kappa^{5}\right)$.

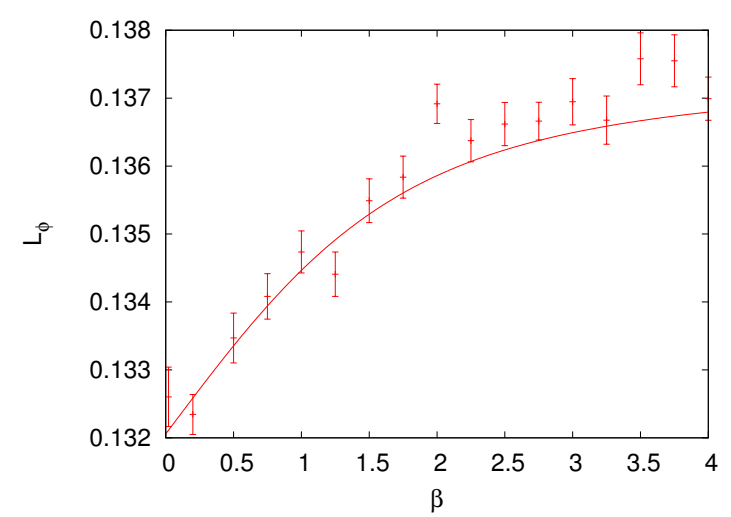

Figure 2: $L_{\phi}$ at fixed $\kappa=0.15$ as function of $\beta_{p l}$ for $\lambda=0.1$ compared with the hopping expansion with included dependence on $\beta_{p l}$ up to $O\left(\kappa^{5}\right)$.

\section{The large $\lambda$ limit}

When $\lambda$ becomes arbitrarily large, $M_{x}$ is frozen to 1 , or in other words, the Brout-EnglertHiggs mode becomes infinitely massive. We are then left with compact variables of integration in the original formulation $\left(\theta_{x}\right.$ and $\left.A_{x, \hat{v}}\right)$ and the Fourier expansions described before lead to expressions of the partition function in terms of discrete sums.

As explained in Ref. [11], these sums can be formulated in a compact way using tensorial notations. The partition function can be written as

$$
Z=\left(I_{0}\left(\beta_{p l}\right) I_{0}\left(2 \kappa_{s}\right) I_{0}\left(2 \kappa_{\tau}\right)\right)^{V} \operatorname{Tr}\left[\prod_{s, \tau, \square} A_{m_{\text {above }} m_{\text {below }}}^{(s)} A_{m_{\text {left }} m_{\text {right }}}^{(\tau)} B_{m_{1} m_{2} m_{3} m_{4}}^{(\square)}\right],
$$

where the $A$ tensors are associated with the spatial and temporal links and $B$ tensors with the plaquettes. $A$ and $B$ tensors are defined in terms of the ratios of Bessel functions $t_{n}(z)=I_{n}(z) / I_{0}(z)$, see Ref. [3] for details.

The traces can also be expressed in terms of a transfer matrix $\mathbb{T}$ which can be constructed by defining the following tensors:

$$
\begin{aligned}
\mathbb{B}_{\left(m_{1}, m_{2}, \ldots m_{N_{s}}\right)\left(m_{1}^{\prime}, m_{2}^{\prime} \ldots N_{s}^{\prime}\right)} & =t_{m_{1}}\left(2 \kappa_{\tau}\right) \delta_{m_{1}, m_{1}^{\prime}} t_{m_{1}}\left(\beta_{p l}\right) t_{\left|m_{1}-m_{2}\right|}\left(2 \kappa_{\tau}\right) \delta_{m_{2}, m_{2}^{\prime}} t_{m_{2}}\left(\beta_{p l}\right) \\
& \times t_{\left|m_{2}-m_{3}\right|}\left(2 \kappa_{\tau}\right) \ldots t_{m_{N_{s}}}\left(\beta_{p l}\right) t_{m_{N_{s}}}\left(2 \kappa_{\tau}\right) . \\
\mathbb{A}_{\left(m_{1}, m_{2}, \ldots m_{N_{s}}\right)\left(m_{1}^{\prime}, m_{2}^{\prime} \ldots m_{N_{s}}^{\prime}\right)} & =t_{\left|m_{1}-m_{1}^{\prime}\right|}\left(2 \kappa_{s}\right) t_{\left|m_{2}-m_{2}^{\prime}\right|}\left(2 \kappa_{s}\right) \ldots t_{\left|m_{N_{s}}-m_{N_{s}}^{\prime}\right|}\left(2 \kappa_{s}\right)
\end{aligned}
$$

(this definition implies open boundary conditions, $m=0$ at both ends). In this case, the chemical potential has completely disappeared. If we had chosen different $m$ 's at the end allowing a total charge $Q$ inside the interval, we would have an additional factor $\exp (\mu Q)$.

Since $\mathbb{B}$ is diagonal, real and positive, the symmetric transfer matrix can be defined as

$$
\mathbb{T}=\sqrt{\mathbb{B}} \mathbb{A} \sqrt{\mathbb{B}} \quad \text { and } \quad Z=\left(I_{0}\left(\beta_{p l}\right) I_{0}\left(2 \kappa_{s}\right) I_{0}\left(2 \kappa_{\tau}\right)\right)^{V} \operatorname{Tr}\left[\mathbb{T}^{N_{\tau}}\right] .
$$

The $\mathbb{A}$ and $\mathbb{B}$ matrices can be constructed by a recursive blocking method similar to those discussed in Ref. [11]. 
In the case where both $\lambda$ and $\beta_{p l}$ are infinite this model corresponds to the two-dimensional classical $O(2)$ model. A Hamiltonian formulation of the model suitable for quantum simulation on optical lattice has been proposed by some of the present authors in Ref. [12].

\section{The time continuum limit, energy spectrum and Bose-Hubbard model implementation}

We construct the time continuum limit of the transfer matrix $\mathbb{T}$ by taking the limit $1<<\beta_{p l}<<$ $\kappa_{\tau}$ and keeping the open boundary conditions ( $m=0$ at both ends). At leading order in the inverse of these large parameters, the eigenvalues of $\mathbb{T}$ are

$$
\begin{aligned}
\lambda_{\left(m_{1}, m_{2}, \ldots m_{N_{s}}\right)} & =1-\frac{1}{2}\left[\frac{1}{\beta_{p l}}\left(m_{1}^{2}+m_{2}^{2}+\ldots+m_{N_{s}}^{2}\right)\right. \\
& \left.+\frac{1}{2 \kappa_{\tau}}\left(m_{1}^{2}+\left(m_{2}-m_{1}\right)^{2}+\ldots+\left(m_{N_{s}}-m_{N_{s}-1}\right)^{2}+m_{N_{s}}^{2}\right)\right]
\end{aligned}
$$

We set the scale with the gap energy $\tilde{U}_{P}$ and relate it to the other energy scales as:

$$
\tilde{U}_{P} \equiv \frac{1}{a \beta_{p l}}, \quad \tilde{Y} \equiv \frac{1}{2 \kappa_{\tau} a}=\frac{\beta_{p l}}{2 \kappa_{\tau}} \tilde{U}_{P}, \quad \tilde{X} \equiv \sqrt{2} \beta_{p l} \kappa_{s} \tilde{U}_{P} .
$$

We can now derive an expression for the Hamiltonian in the spin-1 approximation where the plaquette quantum number $m$ takes values \pm 1 and 0 only. The effect of $\kappa_{s}$ can be included by linearization. The final form of the Hamiltonian $\bar{H}$ for $1<<\beta_{p l}<<\kappa_{\tau}$ is

$$
\bar{H}=\frac{\tilde{U}_{P}}{2} \sum_{i}\left(\bar{L}_{(i)}^{z}\right)^{2}+\frac{\tilde{Y}}{2} \sum_{i}^{\prime}\left(\bar{L}_{(i)}^{z}-\bar{L}_{(i+1)}^{z}\right)^{2}-\tilde{X} \sum_{i} \bar{L}_{(i)}^{x},
$$

where $\sum_{i}^{\prime}$ is a short notation to include the single terms at the two ends as in Eq. (5.1), i.e. besides $\left(\bar{L}_{(1)}^{z}-\bar{L}_{(2)}^{z}\right)^{2},\left(\bar{L}_{(2)}^{z}-\bar{L}_{(3)}^{z}\right)^{2}, \ldots,\left(\bar{L}_{\left(N_{s}-1\right)}^{z}-\bar{L}_{\left(N_{s}\right)}^{z}\right)^{2}$ terms this sum contains $\left(\bar{L}_{(1)}^{z}\right)^{2}$ and $\left(\bar{L}_{\left(N_{s}\right)}^{z}\right)^{2}$.

A generic two-species Bose-Hubbard Hamiltonian for a linear optical lattice realization of the Abelian-Higgs model has the form [3]:

$$
\begin{aligned}
& \mathscr{H}=-\sum_{\langle i j\rangle}\left(t_{a} a_{i}^{\dagger} a_{j}+t_{b} b_{i}^{\dagger} b_{j}+\text { h.c. }\right)-\sum_{i, \alpha}\left(\mu_{a+b}+\Delta_{\alpha}\right) n_{i}^{\alpha} \\
& +\sum_{i, \alpha} \frac{U_{\alpha}}{2} n_{i}^{\alpha}\left(n_{i}^{\alpha}-1\right)+W \sum_{i} n_{i}^{a} n_{i}^{b}+\sum_{\langle i j\rangle \alpha} V_{\alpha} n_{i}^{\alpha} n_{j}^{\alpha}-\frac{t_{a b}}{2} \sum_{i}\left(a_{i}^{\dagger} b_{i}+b_{i}^{\dagger} a_{i}\right)
\end{aligned}
$$

with $\alpha=a, b$ indicating two different species and with $n_{i}^{a}=a_{i}^{\dagger} a_{i}$ and $n_{i}^{b}=b_{i}^{\dagger} b_{i}$. In the limit where $U_{a}=U_{b}=U$ and $W$ and $\mu_{a+b}=(3 / 2) U$ are much larger than any other energy scale, we have the condition $n_{i}^{a}+n_{i}^{b}=2$ for the low energy sector. The three states $|2,0\rangle,|1,1\rangle$ and $|0,2\rangle$ satisfy this condition and correspond to the three states of the spin-1 projection considered above.

At the second-order degenerate perturbation theory this Hamiltonian can be rewritten in terms of the angular momentum operators:

$$
\begin{aligned}
\mathscr{H}_{e f f} & =\left(\frac{V_{a}}{2}-\frac{t_{a}^{2}}{U}+\frac{V_{b}}{2}-\frac{t_{b}^{2}}{U}\right) \sum_{\langle i j\rangle} L_{i}^{z} L_{j}^{z}+\frac{-t_{a} t_{b}}{U} \sum_{\langle i j\rangle}\left(L_{i}^{+} L_{j}^{-}+L_{i}^{-} L_{j}^{+}\right)+(U-W) \sum_{i}\left(L_{i}^{z}\right)^{2} \\
& +\left[\left(\frac{p n}{2} V_{a}+\Delta_{a}-\frac{p(n+1) t_{a}^{2}}{U}\right)-\left(\frac{p n}{2} V_{b}+\Delta_{b}-\frac{p(n+1) t_{b}^{2}}{U}\right)\right] \sum_{i} L_{i}^{z}-t_{a b} \sum_{i} L_{i}^{x},
\end{aligned}
$$


where $p$ is the number of neighbors and $n$ is the occupation ( $p=2, n=2$ in the case under consideration). $\hat{L}$ is the angular momentum operator in the representation $n / 2$.

By imposing $t_{a}=t_{b}=0, V_{a}=V_{b}=-\tilde{Y} / 2$ and $t_{a b}=\tilde{X}$ we match the two-species Bose-Hubbard Hamiltonian (5.5) to the one given in Eq. (5.3). In Fig. 3 we show the spectra of the Abelian-Higgs model obtained with the tensor renormalization group method and the two-species Bose-Hubbard model obtained with exact diagonalization for a system of two (left) and four (right) sites.
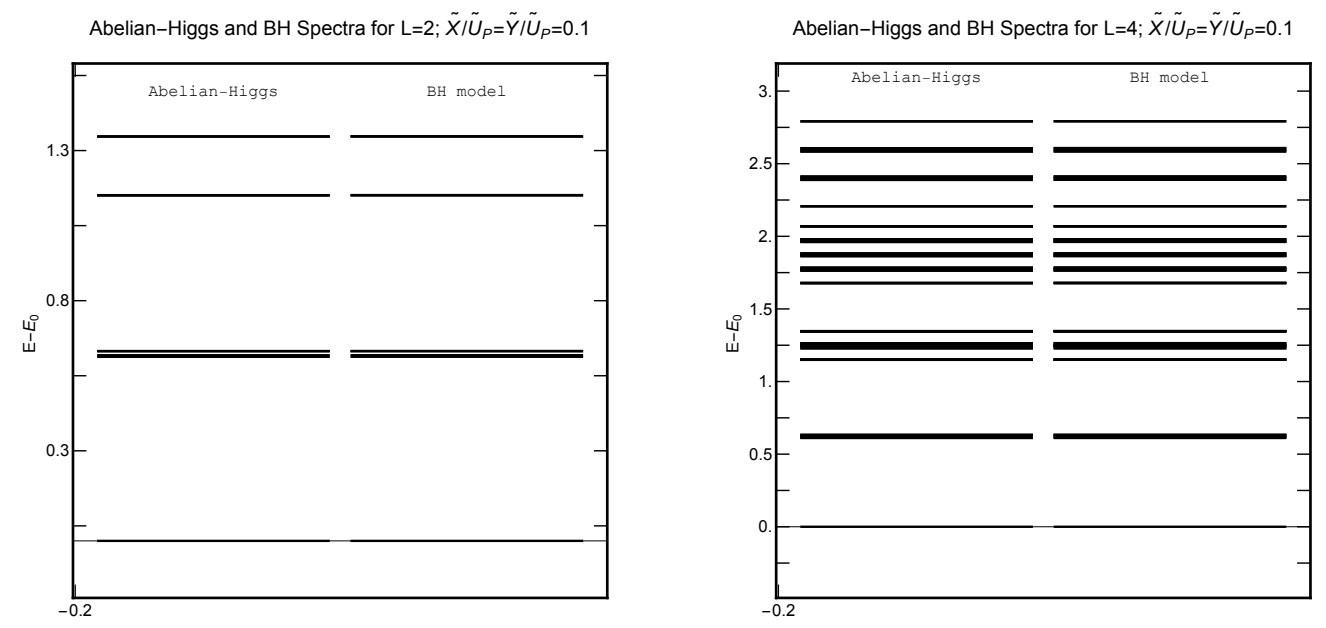

Figure 3: Abelian-Higgs model with $\tilde{X} / \tilde{U}_{P}=0.1, \tilde{Y} / \tilde{U}_{P}=0.1$ and the corresponding Bose-Hubbard spectra for $L=2$ (left) and $L=4$ (right).

The last term in the Hamiltonian, Eq. (5.4), interchanges the species index and thus rules out implementing this Hamiltonian using mixtures of two different types of atoms. It could be realized with a single atomic species on a ladder structure with $a$ and $b$ corresponding to the two legs of the ladder. For the experimental implementation, an attractive nearest neighbor interaction $\left(V_{a}=V_{b}=-\tilde{Y} / 2\right)$ can be obtained by using cold dipolar atoms or molecules, with dipole moments aligned along the ladder and with inter-rung distance such that the rapidly decaying dipoledipole interaction between next-nearest-neighbors can be neglected. Alternatively, the two boson species in the Bose-Hubbard Hamiltonian could correspond to two hyperfine states of the same atom species, provided these two states support Raman transitions between them so that the species conversion term can be created.

\section{Conclusions}

With the remarkable experimental progress in the field of ultra-cold atoms trapped in optical lattices, and the unprecedented levels of control and tunability of their interactions, a wide range of quantum many-body lattice Hamiltonians can be realized and studied. We have proposed a twospecies Bose-Hubbard model that may be used as a quantum simulator for the Abelian-Higgs model in the case of frozen radial mode in $1+1$ dimensions. The gauge invariance is built-in and thus does not need to be achieved via fine-tuning, and the correspondence between the proposed BoseHubbard Hamiltonian and the Abelian-Higgs model can be checked quantitatively. We demonstrate the matching between the two models by calculating the spectra for small systems. 


\section{Acknowledgments}

This research was supported in part by the Department of Energy under Award Numbers DOE grant DE-FG02-05ER41368, DE-SC0010114 and DE-FG02-91ER40664, the NSF under grants DMR-1411345 and PHY11-25915 and by the Army Research Office of the Department of Defense under Award Number W911NF-13-1-0119. We also acknowledge helpful discussions with the participants of the workshops INT-15-1 "Frontiers in Quantum Simulation with Cold Atoms" at INT, Seattle, "Understanding Strongly Coupled Systems in High Energy and Condensed Matter Physics" and "Ultra-Cold Quantum Matter with Atoms and Molecules" at the Aspen Center for Physics.

\section{References}

[1] U.-J. Wiese, Ultracold Quantum Gases and Lattice Systems: Quantum Simulation of Lattice Gauge Theories, Annalen Phys. 525 (2013) 777-796, [arXiv:1305.1602].

[2] E. Zohar, J. I. Cirac, and B. Reznik, Quantum Simulations of Lattice Gauge Theories using Ultracold Atoms in Optical Lattices, arXiv:1503.02312.

[3] A. Bazavov, Y. Meurice, S.-W. Tsai, J. Unmuth-Yockey, and J. Zhang, Gauge-invariant implementation of the abelian-higgs model on optical lattices, Phys. Rev. D 92 (Oct, 2015) 076003.

[4] L. Tagliacozzo, A. Celi, A. Zamora, and M. Lewenstein, Optical Abelian Lattice Gauge Theories, Annals Phys. 330 (2013) 160-191, [arXiv:1205. 0496].

[5] E. Zohar and B. Reznik, Confinement and lattice QED electric flux-tubes simulated with ultracold atoms, Phys.Rev.Lett. 107 (2011) 275301, [arXiv:1108.1562].

[6] E. Zohar, J. I. Cirac, and B. Reznik, Simulating Compact Quantum Electrodynamics with ultracold atoms: Probing confinement and nonperturbative effects, Phys.Rev.Lett. 109 (2012) 125302, [arXiv: 1204.6574$]$.

[7] K. Kasamatsu, I. Ichinose, and T. Matsui, Atomic Quantum Simulation of the Lattice Gauge-Higgs Model: Higgs Couplings and Emergence of Exact Local Gauge Symmetry, Phys.Rev.Lett. 111 (2013), no. 11 115303, [arXiv:1212.4952].

[8] Y. Kuno, K. Kasamatsu, Y. Takahashi, I. Ichinose, and T. Matsui, Real-time dynamics and proposal for feasible experiments of lattice gauge-Higgs model simulated by cold atoms, New J. Phys. 17 (2015), no. 6 063005, [arXiv:1412.7605].

[9] H. Vairinhos and P. de Forcrand, Lattice gauge theory without link variables, JHEP 12 (2014) 038, [arXiv:1409.8442].

[10] J. Heitger, Numerical simulations of gauge-higgs models on the lattice, 1997. (Ph. D. thesis).

[11] Y. Liu, Y. Meurice, M. P. Qin, J. Unmuth-Yockey, T. Xiang, Z. Y. Xie, J. F. Yu, and H. Zou, Exact blocking formulas for spin and gauge models, Phys. Rev. D 88 (Sep, 2013) 056005.

[12] H. Zou, Y. Liu, C.-Y. Lai, J. Unmuth-Yockey, L.-P. Yang, A. Bazavov, Z. Y. Xie, T. Xiang, S. Chandrasekharan, S.-W. Tsai, and Y. Meurice, Progress towards quantum simulating the classical O(2) model, Phys. Rev. A 90 (Dec, 2014) 063603. 